\title{
Long-term neutrino afterglows from gamma-ray bursts
}

\author{
Z. Li, Z. G. Dai, and T. Lu \\ Department of Astronomy, Nanjing University, Nanjing 210093, PR China \\ Received 23 April 2002 / Accepted 22 August 2002

\begin{abstract}
It is widely believed that multiwavelength afterglows of gamma-ray bursts (GRBs) originate from relativistic blast waves. We here show that in such blast waves, a significant fraction of the energy of shock-accelerated protons would be lost due to pion production by interactions with afterglow photons. This could lead to long-term production of $10^{16}-10^{18} \mathrm{eV}$ neutrinos and sub-TeV $\gamma$-rays that accompany usual afterglows, provided that the protons are accelerated to $10^{19} \mathrm{eV}$ in the blast waves.
\end{abstract}

Key words. acceleration of particles - elementary particles - gamma-rays: bursts

\section{Introduction}

In the standard fireball shock model, GRBs and their afterglows are produced due to the dissipation of kinetic energy of relativistic ejecta with a Lorentz factor of $\gamma_{0} \sim 100-1000$, the so-called "fireball", released from an unknown central engine (see Piran 1999; van Paradijs et al. 2000; Mészáros 2002 for detailed reviews). In this model, the shocks, resulting from the collisions between shells with different Lorentz factors in the ejecta (internal shocks) or between the ejecta and the surrounding medium (external shocks), accelerate the electrons responsible for prompt gamma-rays in GRBs and for X-ray, optical and radio emission of afterglows. The same shocks should also accelerate protons. It is pointed out that the physical conditions in the shocks allow protons to be accelerated to $\epsilon_{\mathrm{p}} \sim 10^{20} \mathrm{eV}$ (Waxman 1995a; Vietri 1995; Dermer \& Humi 2001. But see Stecker 2000; Mannheim 2000; Scully \& Stecker 2002). Furthermore, the spectrum and flux of ultra-high energy cosmic rays (UHECRs) are consistent with those expected from Fermi acceleration of protons in GRBs (Waxman 1995b), suggesting a common origin of GRBs and UHECRs. This picture will lead obviously to high energy neutrinos produced by $\pi^{+}$created in photo-pion interactions between the accelerated protons in the internal or external shocks and synchrotron photons from electrons accelerated by the same shocks (Waxman \& Bahcall 1997, 2000; Halzen 1998; Vietri 1998a,b; Dai \& Lu 2001; see Waxman 2001 for a review). This $p \gamma$ reaction has a peak at the energy threshold of the photo-meson $\Delta$-resonance, i.e. $\epsilon_{\gamma} \epsilon_{\mathrm{p}}=0.2 \mathrm{GeV}^{2} \gamma^{2}$, and the resulting neutrinos receive $\sim 5 \%$ of the proton energy from the decay of charged pions and muons. This leads to neutrinos of $\sim 10^{14} \mathrm{eV}$ for prompt gammarays from internal shocks (Waxman \& Bahcall 1997; Halzen 1998), while a UV/optical flash from the external reverse shock implies higher energy neutrinos (e.g., $\sim 10^{18} \mathrm{eV}$ ) (Waxman \& Bahcall 2000). Vietri (1998a, 1998b) studied the neutrino production in the case of external forward shocks, and pointed

Send offprint requests to: $\mathrm{Z}$. Li, e-mail: lizhuo@nju.edu.cn out that the neutrino energy may exceed $10^{19} \mathrm{eV}$. Waxman \& Bahcall (1999) argued that the contributions of external shocks to the neutrino flux is small.

In recent years, the standard afterglow model, which is successful in interpreting observations, has been developed (Mészáros \& Rees 1997; Sari et al. 1998; Wijers \& Galama 1999). In this paper, we re-investigate the case of external shock neutrinos based on the present detailed afterglow model. We derive the maximum energy that protons could reach by Fermi acceleration and the efficiency of energy loss from accelerated protons to pions in the forward shock. By considering the cooling of secondary particles, $\pi^{+}$and $\mu^{+}$, we derive the spectrum and the flux of the resulting neutrinos. We find that at late times, e.g., $\sim 1$ day after the GRB trigger, the forward shock may be able to produce an intense neutrino signal, even though the flux is smaller at earlier times, as stated by Waxman \& Bahcall (1999).

We discuss in Sect. 2 the standard afterglow model, including the dynamics and synchrotron radiation of the blast wave, and in Sect. 3 the proton acceleration by the same shock. In Sect. 4 we discuss the neutrino production rate from the shockaccelerated protons and the maximum energy that neutrinos may achieve. The neutrino spectrum, flux and detectability are derived in Sect. 5. Conclusions and discussion are given in Sect. 6.

\section{Dynamics and emissions of afterglows}

The fireball shock model suggests that after the prompt burst from internal shocks, the ejecta with total kinetic energy $E$ continues to expand and drives a relativistic blast wave into the surrounding medium. Once the shocked medium gets an energy similar to $E$, the blast wave will enter the BlandfordMcKee (1976) self-similar evolution, where the radius $r$ and the Lorentz factor $\gamma$ evolve with observer time as (Wijers \& Galama 1999)

$r=4.07 \times 10^{17}\left(E_{53} / n_{0}\right)^{1 / 4} t_{\mathrm{d}}^{1 / 4} \mathrm{~cm}$, 


$$
\gamma=8.87\left(E_{53} / n_{0}\right)^{1 / 8} t_{\mathrm{d}}^{-3 / 8},
$$

where $n$ is the baryon number density of the medium, and $t_{\mathrm{d}}=$ $t / 1$ day. Unless stated otherwise, the useful form $Q=10^{x} Q_{x}$ has been used in this paper.

Let $\xi_{\mathrm{e}}$ and $\xi_{\mathrm{B}}$ be the fractions of the post-shock energy density $U=4 \gamma^{2} n m_{\mathrm{p}} c^{2}$ (in the comoving frame with the shocked material) that are carried by electrons and magnetic fields respectively. The characteristic electron Lorentz factor (in the comoving frame of the shocked material) is $\gamma_{\mathrm{m}} \simeq \xi_{\mathrm{e}} \gamma m_{\mathrm{p}} / m_{\mathrm{e}}$, where the post-shock energy per proton is $\gamma m_{\mathrm{p}} c^{2}$. The strength of the magnetic field in the comoving frame is

$B=0.34 \xi_{\mathrm{B},-2}^{1 / 2} E_{53}^{1 / 8} n_{0}^{3 / 8} t_{\mathrm{d}}^{-3 / 8} \mathrm{G}$.

From the above equations, we can calculate the evolution of synchrotron spectrum from the blast wave. The characteristic energy of synchrotron photons from electrons with Lorentz factor $\gamma_{\mathrm{m}}$ is

$\epsilon_{\mathrm{m}}^{\mathrm{ob}} \simeq \hbar \gamma \gamma_{\mathrm{m}}^{2} \frac{e B}{m_{\mathrm{e}} c}=0.09 \xi_{\mathrm{e},-1}^{2} \xi_{\mathrm{B},-2}^{1 / 2} E_{53}^{1 / 2} t_{\mathrm{d}}^{-3 / 2} \mathrm{eV}$,

where and hereafter we denote the particle energy in the observer frame with superscript "ob", and particle energy measured at the comoving frame without superscript. The characteristic energy of synchrotron photons from electrons with the cooling time, $6 \pi m_{\mathrm{e}} c / \sigma_{\mathrm{T}} \gamma_{\mathrm{c}} B^{2}$, which equals the blast wave expansion time $(\sim r / \gamma c)$, is given by

$\epsilon_{\mathrm{c}}^{\mathrm{ob}} \simeq \hbar \gamma \gamma_{\mathrm{c}}^{2} \frac{e B}{m_{\mathrm{e}} c}=3 \xi_{\mathrm{B},-2}^{-3 / 2} n_{0}^{-1} E_{53}^{-1 / 2} t_{\mathrm{d}}^{-1 / 2} \mathrm{eV}$,

and the specific luminosity $L_{\epsilon}=\mathrm{d} L / \mathrm{d} \epsilon^{\mathrm{ob}}$ at the peak is

$L_{\max } \simeq(2 \pi \hbar)^{-1} \gamma \frac{e^{3} B}{m_{\mathrm{e}} c^{2}} N_{\mathrm{e}} \simeq 5 \times 10^{58}\left(\xi_{\mathrm{B},-2} n_{0}\right)^{1 / 2} E_{53} \mathrm{~s}^{-1}$,

where $N_{\mathrm{e}}=\frac{4}{3} \pi r^{3} n$ is the total number of the swept-up electrons. Equation (6) implies that the peak specific luminosity is a time-independent constant. The electrons are accelerated by the shock to a power-law energy distribution, $\mathrm{d} N_{\mathrm{e}} / \mathrm{d} \gamma_{\mathrm{e}} \propto \gamma_{\mathrm{e}}^{-p}$ for $\gamma_{\mathrm{e}}>\gamma_{\mathrm{m}}$, where $p \simeq 2.2$ is fitted to the observations of afterglows. We take $p=2$ for simplicity here. After $t \sim 1$ hour, $\epsilon_{\mathrm{c}}^{\mathrm{ob}}>\epsilon_{\mathrm{m}}^{\mathrm{ob}}$. In this case, the specific luminosity $L_{\epsilon}$ peaks at $\epsilon_{\mathrm{m}}^{\mathrm{ob}}$, $L_{\text {max }}=L_{\mathrm{m}}$, and the synchrotron spectrum is a broken power law (Sari et al. 1998) as: $L_{\epsilon}=L_{\mathrm{m}}\left(\epsilon / \epsilon_{\mathrm{m}}\right)^{-1 / 2}$ for $\epsilon_{\mathrm{m}}<\epsilon<\epsilon_{\mathrm{c}}$ and $L_{\epsilon} \propto \epsilon^{-1}$ for $\epsilon>\epsilon_{\mathrm{c}}$. Note that in the above equations, the typical parameter values have been adopted as $\xi_{\mathrm{e}}=10^{-1} \xi_{\mathrm{e},-1}$, $\xi_{\mathrm{B}}=10^{-2} \xi_{\mathrm{B},-2}, E=10^{53} E_{53}$ erg and $n=1 n_{0} \mathrm{~cm}^{-3}$, which are inferred from some afterglow observations (e.g. Wijers \& Galama 1999; Granot et al. 1999).

\section{High-energy proton production}

Following the analysis of Waxman (1995a) in the case of internal shocks, we investigate the physical condition in external forward shocks for high energy protons production. The typical Fermi acceleration time is $t_{\mathrm{a}}=f R_{\mathrm{L}} / c$, where the Larmor radius is given by $R_{\mathrm{L}}=\epsilon_{\mathrm{p}} / e B$ and $f$ is of order unity (Hillas 1984). The protons are available for acceleration only within a comoving expansion time $t_{\mathrm{d}} \sim r / \gamma c$. This leads to the requirement that $t_{\mathrm{a}}<t_{\mathrm{d}}$. From Eqs. (1)-(3), we have

$\epsilon_{\mathrm{p}}^{\mathrm{ob}}<4 \times 10^{19} \xi_{\mathrm{B},-2}^{1 / 2} E_{53}^{3 / 8} n_{0}^{1 / 8} t_{\mathrm{d}}^{-1 / 8} \mathrm{eV}$.

If a magnetic field is nearly in equipartition (i.e., $\xi_{\mathrm{B}} \sim 1$ ), the protons can be accelerated to well above $10^{20} \mathrm{eV}$. However, fits of afterglow observations give a typical value $\xi_{\mathrm{B}}=0.01$, showing that the magnetic fields are too weak to accelerate protons up to $10^{20} \mathrm{eV}$ in the blast waves. This upper limit is somewhat robust since its dependence on the medium density and the time $\left(\propto n^{1 / 8} t^{-1 / 8}\right)$ is very weak.

The proton acceleration is affected by energy loss due to synchrotron radiation. The requirement that the proton synchrotron cooling time, $t_{\mathrm{p}, \text { syn }}=\left(6 \pi m_{\mathrm{p}}^{4} c^{3} / \sigma_{\mathrm{T}} m_{\mathrm{e}}^{2}\right) \epsilon_{\mathrm{p}}^{-1} B^{-2}$, should be larger than the acceleration time $t_{\mathrm{a}}$ is

$\epsilon_{\mathrm{p}}^{\mathrm{ob}}<3.2 \times 10^{21} \xi_{\mathrm{B},-2}^{-1 / 4} E_{53}^{1 / 16} n_{0}^{-5 / 16} t_{\mathrm{d}}^{-3 / 16} \mathrm{eV}$.

The proton acceleration is also limited by energy loss due to interaction with afterglow photons. In the next section, we show that, provided a typical medium density $n_{0}=1$, the fraction of energy loss of a proton in the expansion time $t_{\mathrm{d}}$ is usually not larger than one, which implies that the limit from energy loss due to photo-pion interaction is a less stringent constraint than that from $t_{\mathrm{a}}<t_{\mathrm{d}}$. Thus, the maximum energy of protons comes from Eq. (7), $\epsilon_{\mathrm{p}, \max }^{\mathrm{ob}} \simeq 4 \times 10^{19} \mathrm{eV}$.

Gallant \& Achterberg (1999) studied the shockacceleration, also called first order Fermi-acceleration, in GRBs and argued that it may be impossible for protons to be shock-accelerated to above $10^{18.5} \mathrm{eV}$ in an ultra-relativistic blast wave which is driven by the fireball ejecta into a typical interstellar medium. However we still expect protons to be accelerated to ultra-high energy in the blast waves by second order Fermi-acceleration, where the protons gain energy from the scattering at plasma waves (e.g. Dermer \& Humi 2001).

\section{Neutrino production}

Denoting the photon number density in the comoving frame of the shocked medium by $n_{\gamma}(\epsilon) \mathrm{d} \epsilon$ and following Waxman \& Bahcall (1997), we can write the fractional energy-loss rate of a proton with energy $\epsilon_{\mathrm{p}}$ due to pion production,

$$
\begin{aligned}
t_{\pi}^{-1}\left(\epsilon_{\mathrm{p}}\right) & \equiv-\frac{1}{\epsilon_{\mathrm{p}}} \frac{\mathrm{d} \epsilon_{\mathrm{p}}}{\mathrm{d} t} \\
& =\frac{1}{2 \gamma_{\mathrm{p}}^{2}} c \int_{\epsilon_{0}}^{\infty} \mathrm{d} \epsilon \sigma_{\pi}(\epsilon) \xi(\epsilon) \epsilon \int_{\epsilon / 2 \gamma_{\mathrm{p}}}^{\infty} \mathrm{d} x x^{-2} n(x),
\end{aligned}
$$

where $\gamma_{\mathrm{p}}=\epsilon_{\mathrm{p}} / m_{\mathrm{p}} c^{2}, \sigma_{\pi}(\epsilon)$ is the cross section for pion production for a photon with energy $\epsilon$ in the proton rest frame, $\xi(\epsilon)$ is the average fraction of energy lost to the pion, $\epsilon_{0}=0.15 \mathrm{GeV}$ is the threshold energy, and the proton number density is related to the observed specific luminosity by $n(x)=L_{\epsilon}(\gamma x) /\left(4 \pi r^{2} c \gamma x\right)$. Because of the $\Delta$-resonance, the photo-meson production for $\epsilon_{0} / 2 \epsilon_{\mathrm{c}} \ll \gamma_{\mathrm{p}}<\epsilon_{0} / 2 \epsilon_{\mathrm{m}}$ is dominated by interaction with photons in the energy range $\epsilon_{\mathrm{m}}<$ $\epsilon \ll \epsilon_{\mathrm{c}}$, where $L_{\epsilon} \propto \epsilon^{-1 / 2}$. For this photon spectrum, Eq. (9) leads to

$t_{\pi}^{-1}\left(\epsilon_{\mathrm{p}}\right) \simeq \frac{2^{3 / 2}}{2.5} \frac{L_{\mathrm{m}}}{4 \pi r^{2} \gamma}\left(\frac{\epsilon_{\mathrm{peak}}}{\gamma_{\mathrm{p}} \epsilon_{\mathrm{m}}}\right)^{-1 / 2} \frac{\sigma_{\text {peak }} \xi_{\text {peak }} \Delta \epsilon}{\epsilon_{\text {peak }}}$. 
Here, $\sigma_{\text {peak }} \simeq 5 \times 10^{-28} \mathrm{~cm}^{2}$ and $\xi_{\text {peak }} \simeq 0.2$ at the resonance $\epsilon=\epsilon_{\text {peak }}=0.3 \mathrm{GeV}$, and $\Delta \epsilon \simeq 0.2 \mathrm{GeV}$ is the peak width. The fraction of energy loss of a proton with observed energy $\epsilon_{\mathrm{p}}^{\mathrm{ob}}$ by pion production, $f_{\pi}\left(\epsilon_{\mathrm{p}}^{\mathrm{ob}}\right)$, is estimated by $t_{\pi}^{-1}\left(\epsilon_{\mathrm{p}}\right)$ times the comoving expansion time of the shocked material $(\sim r / \gamma c)$. Thus, according to Eqs. (1), (2), (4) and (6), we obtain

$f_{\pi}\left(\epsilon_{\mathrm{p}}^{\mathrm{ob}}\right) \approx 0.07 \xi_{\mathrm{e},-1} \xi_{\mathrm{B},-2}^{3 / 4} E_{53}^{5 / 8} n_{0}^{9 / 8} t_{\mathrm{d}}^{1 / 8}\left(\epsilon_{\mathrm{p}, 19}^{\mathrm{ob}}\right)^{1 / 2}$.

Equation (11) is valid for protons in the energy range

$$
\begin{aligned}
& 6 \times 10^{17} \xi_{\mathrm{B},-2}^{3 / 2} E_{53}^{3 / 4} n_{0}^{3 / 4} t_{\mathrm{d}}^{-1 / 4} \mathrm{eV}<\epsilon_{\mathrm{p}}^{\mathrm{ob}} \\
< & 5 \times 10^{19} \xi_{\mathrm{e},-1}^{-2} \xi_{\mathrm{B},-2}^{-1 / 2} E_{53}^{-1 / 4} n_{0}^{-1 / 4} t_{\mathrm{d}}^{3 / 4} \mathrm{eV} .
\end{aligned}
$$

At lower energy, protons interact mainly with photons in the energy range $\epsilon>\epsilon_{\mathrm{c}}$, where $L_{\epsilon} \propto \epsilon^{-1}$, and $f_{\pi} \propto \epsilon_{\mathrm{p}}^{\mathrm{ob}}$. Equations (11) and (12) imply that the fraction of energy loss, $f_{\pi \mathrm{b}}$, at the "break" energy $\epsilon_{\mathrm{pb}}^{\mathrm{ob}}=6 \times 10^{17} \xi_{\mathrm{B},-2}^{3 / 2} E_{53}^{3 / 4} n_{0}^{3 / 4} t_{\mathrm{d}}^{-1 / 4} \mathrm{eV}$, is a time-independent constant,

$f_{\pi \mathrm{b}} \approx 0.02 \xi_{\mathrm{e},-1} \xi_{\mathrm{B},-2}^{3 / 2} E_{53} n_{0}^{3 / 2}$.

The maximum energy of the resultant neutrinos is analyzed as follows. This energy is first determined by the maximum energy that the accelerated protons achieve. From Eq. (7), the maximum energy of neutrinos is $\sim 2 \times 10^{18} \mathrm{eV}$. This energy is also limited by the energy loss of secondary particles, i.e., pions and muons, because both pions and muons may suffer synchrotron and inverse-Compton (IC) losses before decay (Rachen \& Mészáros 1998). For synchrotron losses of pions and muons to be negligible, their Lorentz factor (in the comoving frame of shocked medium) should not exceed the critical Lorentz factor $\gamma_{\star}$ given by $\gamma_{\star}^{2} \tau_{\star}=\gamma_{\mathrm{p}} t_{\mathrm{p}, \text { syn }}\left(m_{\star} / m_{\mathrm{p}}\right)^{3}$ (Rachen $\&$ Mészáros 1998), where $\star$ refers to either pions or muons, $\tau_{\pi}=2.6 \times 10^{-8} \mathrm{~s}$ and $\tau_{\mu}=2.2 \times 10^{-6} \mathrm{~s}$ are respective lifetimes in their rest frames, and $m_{\star} / m_{\mathrm{p}} \simeq 0.1$ for both pions and muons. Due to the longer lifetime compared to pions, muons suffer greater energy loss, resulting in lower upper limit of neutrino energy. Using Eq. (3), we have (in the observer frame) the critical energy for muons

$\epsilon_{\mu, \text { syn }}^{\mathrm{ob}}=10^{20} \xi_{\mathrm{B},-2}^{-1 / 2} n_{0}^{-1 / 2} \mathrm{eV}$,

which is independent of the total fireball energy $E$ and observer time $t$. For IC loss to be negligible, the critical Lorentz factor is given by $\gamma_{\star}^{2} \tau_{\star}=\gamma_{\mathrm{p}} t_{\mathrm{p}, \text { syn }}\left(m_{\star} / m_{\mathrm{p}}\right)^{3}\left(U_{\mathrm{B}} / U_{\mathrm{ph}}\right)$, where $U_{\mathrm{B}}=\xi_{\mathrm{B}} U$ and $U_{\mathrm{ph}}=\xi_{\mathrm{e}} \eta U$ are the energy densities of the magnetic field and afterglow photons, respectively, and $\eta$ is the fraction of the electron energy that is radiated away. Note that $\eta=\left(\epsilon_{\mathrm{c}} / \epsilon_{\mathrm{m}}\right)^{(2-p) / 2}$ for slow cooling electrons with $\epsilon_{\mathrm{c}}>\epsilon_{\mathrm{m}}$. We take $p \approx 2.2$. $\eta$ is not sensitive to other parameters as $\eta \sim 1$. (In fact, $\eta=1$ shows the strongest IC loss and provides the strongest constraint). Thus, the critical Lorentz factor is $\gamma_{\star}^{\mathrm{IC}} \approx \gamma_{\star}^{\mathrm{syn}}\left(\xi_{\mathrm{B}} / \xi_{\mathrm{e}}\right)^{1 / 2}$, or

$\epsilon_{\mu, \mathrm{IC}}^{\mathrm{ob}}=4 \times 10^{19} \xi_{\mathrm{e},-1}^{-1 / 2} n_{0}^{-1 / 2} \mathrm{eV}$,

which is independent of $\xi_{\mathrm{B}}$. The IC loss is somewhat stronger than the synchrotron loss provided $\xi_{\mathrm{B}} / \xi_{\mathrm{e}}<1$. But, the energy limit from both losses exceeds the maximum proton energy from Eq. (7), so the neutrino production does not suffer the energy losses of secondary particles. The maximum neutrino energy is determined by the maximum proton energy, $\epsilon_{v, \max }^{\mathrm{ob}} \simeq 2 \times 10^{18} \mathrm{eV}$.

\section{Neutrino spectrum, flux and detectability}

The photo-meson interactions include (1) production of $\pi$ mesons: $p \gamma \rightarrow p+\pi^{0}$ and $p \gamma \rightarrow n+\pi^{+}$, and (2) decay of $\pi$ mesons: $\pi^{0} \rightarrow 2 \gamma$ and $\pi^{+} \rightarrow \mu^{+}+v_{\mu} \rightarrow \mathrm{e}^{+}+v_{\mathrm{e}}+\bar{v}_{\mu}+v_{\mu}$. These processes produce neutrinos with energy $\sim 5 \% \epsilon_{\mathrm{p}}$ (Waxman $\&$ Bahcall 1997). Equation (12) implies that for a fixed time, the spectrum of neutrinos below $\epsilon_{v \mathrm{~b}}^{\mathrm{ob}} \approx 3 \times 10^{16} \xi_{\mathrm{B},-2}^{3 / 2} E_{53}^{3 / 4} n_{0}^{3 / 4} t_{\mathrm{d}}^{-1 / 4} \mathrm{eV}$ is harder by one power of the energy than the proton spectrum, and by half a power of the energy at higher energy. Therefore, if the differential spectrum of accelerated protons is a power law form $n\left(\epsilon_{\mathrm{p}}\right) \propto \epsilon_{\mathrm{p}}^{-2}$ (Blandford \& Eichler 1987), the differential neutrino spectrum is $n\left(\epsilon_{v}\right) \propto \epsilon_{v}^{-1}$ below the break and $n\left(\epsilon_{v}\right) \propto \epsilon_{v}^{-3 / 2}$ above the break. Unlike the prompt bursts of neutrinos from either internal or reverse shocks, which has a short duration similar to the GRBs, the neutrino emission from forward shocks last for a longer time due to the long-term forward shocks. Without the detailed knowledge of the evolution of the spectrum of accelerated protons in the forward shocks, it is far from calculating clearly the neutrino fluxes. However, as $\epsilon_{v \mathrm{~b}}^{\mathrm{ob}}\left(\propto t^{-1 / 4}\right)$ and $\epsilon_{\mathrm{p}, \max }^{\mathrm{ob}}\left(\propto t^{-1 / 8}\right)$ do not change much from one day to even weeks, one can expect a time-integrated neutrino spectrum similar to the spectrum at $t=1$ day. For simplicity, the particle energies, hereafter in this section, refer to the value when $t=1$ day, e.g. $\epsilon_{v \mathrm{~b}}^{\mathrm{ob}} \rightarrow \epsilon_{v \mathrm{~b}}^{\mathrm{ob}}\left(t_{\mathrm{d}}=1\right), \epsilon_{\mathrm{pb}}^{\mathrm{ob}} \rightarrow \epsilon_{\mathrm{pb}}^{\mathrm{ob}}\left(t_{\mathrm{d}}=1\right)$, etc.

We derive the neutrino fluxes as follows. Defining by $\bar{f}_{\pi} \equiv$ $\int n\left(\epsilon_{\mathrm{p}}\right) \epsilon_{\mathrm{p}} f_{\pi} \mathrm{d} \epsilon_{\mathrm{p}} / \int n\left(\epsilon_{\mathrm{p}}\right) \epsilon_{\mathrm{p}} \mathrm{d} \epsilon_{\mathrm{p}}$ the fraction of energy loss of all accelerated protons to neutrinos, and considering the neutrino spectrum given in the above paragraph, we obtain, after some calculations,

$\bar{f}_{\pi} \simeq 2 f_{\pi \mathrm{b}} \frac{\left(\epsilon_{\mathrm{p}, \max }^{\mathrm{ob}} / \epsilon_{\mathrm{pb}}^{\mathrm{ob}}\right)^{1 / 2}}{\ln \left(\epsilon_{\mathrm{p}, \max }^{\mathrm{ob}} / \epsilon_{\mathrm{pl}}\right)}$, for $\epsilon_{\mathrm{pl}} \leq \epsilon_{\mathrm{pb}}^{\mathrm{ob}}$

Here $f_{\pi \mathrm{b}}$ is a constant for a certain GRB, therefore this value is mainly bound to $\epsilon_{\mathrm{pl}}$ which is determined by the lower threshold of a detector. In this equation, $\epsilon_{\mathrm{pb}}^{\mathrm{ob}}$ should be replaced by $\epsilon_{\mathrm{pl}}$ for $\epsilon_{\mathrm{pl}} \geq \epsilon_{\mathrm{pb}}^{\mathrm{ob}}$. Taking $\epsilon_{\mathrm{pl}}=\epsilon_{\mathrm{pb}}^{\mathrm{ob}}$, we have $\bar{f}_{\pi} \approx 0.07$.

The present day muon neutrino energy flux due to GRBs is approximately given by $J \approx 0.25(c / 4 \pi) \bar{f}_{\pi} \dot{E} t_{\mathrm{H}}$, where $t_{\mathrm{H}} \approx 10 \mathrm{Gyr}$ is the Hubble time, and $\dot{E}$ is the production rate of UHECRs in GRBs per unit volume. The factor 0.25 here accounts for that about one half of the proton energy is lost to neutral pions which do not produce neutrinos, and about one half of the energy in charged pions is converted to $v_{\mu}$ and

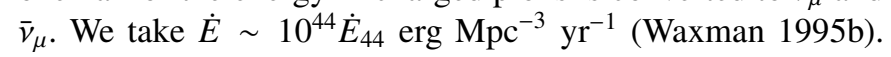
Integrating the specific neutrino flux $\Phi_{v}$ over the neutrino spec$\operatorname{trum}\left(\Phi_{v} \propto\left[\epsilon_{v}^{\mathrm{ob}}\right]^{0}\right.$ below $\epsilon_{v \mathrm{~b}}^{\mathrm{ob}}$, and $\Phi_{v} \propto\left[\epsilon_{v}^{\mathrm{ob}}\right]^{-1 / 2}$ above $\left.\epsilon_{v \mathrm{~b}}^{\mathrm{ob}}\right)$, we have $J=\int \Phi_{\nu} \mathrm{d} \epsilon_{v} \simeq 2\left(\epsilon_{v \mathrm{~b}}^{\mathrm{ob}} \epsilon_{v, \text { max }}^{\mathrm{ob}}\right)^{1 / 2} \Phi_{\nu \mathrm{b}}$. Combining these two equations about $J$ one obtains that the neutrino flux at $\epsilon_{v \mathrm{~b}}^{\mathrm{ob}}$ is

$$
\begin{aligned}
\Phi_{\nu \mathrm{b}} \approx & 2 \times 10^{-18} \frac{\bar{f}_{\pi}}{0.07} \dot{E}_{44} \\
& \times\left(\frac{\epsilon_{\nu \mathrm{b}}^{\mathrm{ob}}}{3 \times 10^{16} \mathrm{eV}} \frac{\epsilon_{v, \max }^{\mathrm{ob}}}{2 \times 10^{18} \mathrm{eV}}\right)^{-1 / 2} \mathrm{~cm}^{-2} \mathrm{~s}^{-1} \mathrm{sr}^{-1}
\end{aligned}
$$

The resulting high-energy neutrinos may be observed by detecting the Cherenkov light emitted by upward moving muons 
produced by neutrino interactions below a detector on the surface of the Earth (Gaisser et al. 1995; Gandhi et al. 1998). Planned $1 \mathrm{~km}^{3}$ detectors of high energy neutrinos include ICECUBE, ANTARES, NESTOR (Halzen 1999) and NuBE (Roy et al. 1999). The probability that a neutrino could produce a high-energy muon in the detector is approximated by $P_{v \rightarrow \mu} \approx 10^{-3}\left(\epsilon_{v} / 10^{16} \mathrm{eV}\right)^{1 / 2}$. We obtain the observed neutrino event rate in a detector,

$$
\begin{aligned}
N_{\text {events }}\left(>\epsilon_{\nu \mathrm{b}}^{\mathrm{ob}}\right) & =2 \pi \int \frac{\Phi_{v}}{\epsilon_{v}^{\mathrm{ob}}} P_{\nu \rightarrow \mu} \mathrm{d} \epsilon_{v}^{\mathrm{ob}} \\
& \approx 2 \pi \Phi_{\nu \mathrm{b}} P_{v \rightarrow \mu}\left(\epsilon_{v \mathrm{~b}}^{\mathrm{ob}}\right) \ln \left(\frac{\epsilon_{v, \mathrm{max}}^{\mathrm{ob}}}{\epsilon_{v \mathrm{~b}}^{\mathrm{ob}}}\right) \\
& \approx 0.04 \frac{\bar{f}_{\pi}}{0.07} \dot{E}_{44}\left(\frac{\epsilon_{v, \mathrm{max}}^{\mathrm{ob}}}{2 \times 10^{18} \mathrm{eV}}\right)^{-1 / 2} \mathrm{~km}^{-2} \mathrm{yr}^{-1}
\end{aligned}
$$

\section{Conclusions and discussion}

After a GRB, the fireball ejecta continues to drive a relativistic blast wave into the surrounding medium. The shock wave accelerates the electrons to give rise to observed afterglow by synchrotron radiation. The protons in the shock are also expected to be Fermi-accelerated at the same time. However, the maximum proton energy may not well exceed $\sim 10^{20} \mathrm{eV}$ based on the typical parameters of afterglows. The interaction of the protons with synchrotron photons leads to a significant fraction of proton energy loss to neutrino production at energy $10^{16}-10^{18} \mathrm{eV}$. Though the synchrotron photon energy density in the blast wave is much lower than in the reverse shock, the efficiency of proton energy loss in the blast wave is still comparable to that in the reverse shock, because of the long expansion time of the blast wave available for energy loss of protons. Since both shocks carry comparable energies (Sari \& Piran 1999), we assume the same production rate of highenergy cosmic rays, $\dot{E}_{44} \simeq 1$ for blast waves as Waxman \& Bahcall (2000) for reverse shocks. Thus, the expected detection rate of muon neutrinos $0.04 \mathrm{~km}^{-2} \mathrm{yr}^{-1}$ is also comparable to the reverse shock. These mean that besides the internal shock and the reverse shock, the blast wave is also one region capable of intense neutrino production.

Waxman \& Bahcall (1999) have estimated a much lower efficiency of the blast wave, i.e. $f_{\pi} \sim 10^{-4}$, using Eq. (4) of Waxman \& Bahcall (1997). However, the parameter values they chose are only valid for the early phase when the external shock just forms. When a set of parameters, which are valid for afterglows one day after the burst, are chosen as follows: $L_{\gamma}=10^{45} \mathrm{erg} / \mathrm{s}, \Delta t=10^{5} \mathrm{~s}, \epsilon_{\gamma \mathrm{b}}^{\mathrm{ob}}=0.1 \mathrm{eV}$ and $\Gamma=10$, their equation gives an efficiency $f_{\pi} \sim 10^{-2}$ in general agreement with ours by orders of magnitude.

It may be that UHECRs cannot be produced in an ultrarelativistic forward shock (Gallant \& Achterberg 1999) during the transition of the shock to the self-similar expansion. However the hard X-rays from the forward shock will interact with the protons accelerated by the reverse shock, yielding a new component of neutrino emission besides those produced by interaction between protons and optical/UV photons from the reverse shock, which is proposed by
Waxman \& Bahcall (2000). At the moment of transition, especially for the photons at energy $\epsilon^{\mathrm{ob}} \gtrsim \epsilon_{\mathrm{m}}^{\mathrm{ob}} \simeq 80 \mathrm{keV}$ (cf. Eq. (4)), the specific luminosity from the forward shock dominates that from the reverse shock, which implies a higher neutrino flux at energy $\epsilon_{v}^{\mathrm{ob}} \lesssim 10^{15}\left(\gamma_{0} / 250\right)\left(\epsilon_{\mathrm{m}}^{\mathrm{ob}} / 100 \mathrm{keV}\right)^{-1} \mathrm{eV}$.

Different models for progenitors of GRBs include different environments: compact object mergers occur in the ISM and the explosions of massive stars in the preexisting stellar wind or denser medium. Dai \& Lu (2001) have found that the neutrino production from reverse shocks in the wind case has a quite different spectrum and 10 times higher detection rate compared with the ISM case, which provides a way to distinguish between GRB progenitor models. In the blast-wave case discussed in this paper, the proton energy loss rate shows a strong dependence on density $n$ (Eq. (11)); we expect much higher fluxes for a denser medium. For $n_{4}=1$ typical of giant molecular clouds, following the analysis in this paper, the energy loss efficiency of a proton at one day after the the GRB trigger is given by $f_{\pi} \approx 8.6 \epsilon_{\mathrm{p}, 18}^{\mathrm{ob}} t_{\mathrm{d}}^{1 / 4}$ for $\epsilon_{\mathrm{p}}^{\mathrm{ob}}<5 \times 10^{18} t_{\mathrm{d}}^{3 / 4} \mathrm{eV}$, implying that protons of energy $\epsilon_{\mathrm{p}}^{\mathrm{ob}}>10^{17} \mathrm{eV}$ lose all their energy to pion production and the neutrino spectrum for $\epsilon_{v}^{\mathrm{ob}}>5 \times 10^{15} \mathrm{eV}$ will trace the proton one. The maximum energy that protons can be accelerated to, which is now confined by the pion production, is $4 \times 10^{18} t_{\mathrm{d}}^{-3 / 4} \mathrm{eV}$. This implies a maximum neutrino energy of $\sim 10^{17} \mathrm{eV}$.

Another consequence of the photo-meson interactions is a sub-TeV afterglow. The decay of neutral pions produced in the blast waves would lead to $\sim 10^{18} \mathrm{eV}$ photons. Such high energy photons would undergo pair production in the afterglows because the optical depth for photons above $\sim 1 \mathrm{TeV}$ is important (Zhang \& Mészáros 2001). So these ultra-high-energy photons would be degraded and leak out as a long-term sub-TeV afterglow, carrying away $\sim 10 \%$ energy of protons, similar to the energy losses to neutrinos. For a GRB at typical redshift $z=1$, the intergalactic diffuse radiation field absorbs photons above $100 \mathrm{GeV}$ (Mannheim et al. 1996), allowing only a multi$\mathrm{GeV}$ afterglow observed on the earth. An EGRET observation of $\mathrm{GeV}$ emission extended to 1.5 hours after the trigger of GRB 940217 (Hurley 1994) has indicated that a high energy spectral component can extend to such high energy band and persist for a long period of time. However, the electron-IC emission in the blast wave can also contribute to this $\mathrm{GeV}$ observation. For the parameters, $n_{0} \simeq \xi_{\mathrm{e},-1} \simeq \xi_{\mathrm{B},-2} \simeq 1$, adopted in this paper, the electron-IC emission is important and plausible to explain the observation (Zhang \& Mészáros 2001). From the afterglow model presented in Sect. 2, we can see that the peak energy in the IC emission spectrum, $\gamma_{\mathrm{c}}^{2} \epsilon_{\mathrm{c}}^{\mathrm{ob}}$ (Sari \& Esin 2001), is still up to $100 \mathrm{GeV}$ even one day after the GRB trigger, but we do not expect GeV IC emission a few days later, while the emission produced by neutral pion decay might still be possible. In principle, the $\mathrm{GeV}$ emission could also be due to the proton synchrotron emission, but it is not important for the parameters adopted here (Zhang \& Mészáros 2001).

Note added in proof: We are very grateful to Charles Dermer for informing us his recent paper (Dermer 2002, ApJ, 574, 65) on discussions of some similar questions. He studied in detail the neutrino production in the external shock model of GRBs. 
Assuming that GRBs are the sources of UHECRs, i.e., protons in the forward shock of a GRB can be accelerated up to $>10^{20} \mathrm{eV}$, and taking a GRB rate density based on the GRB statistics, he carried out numerical calculations on the neutrino production efficiency, spectrum and size distribution, which implies that the neutrino event rate detected from GRBs in a $\mathrm{km}$ scale neutrino detector is likely to be very small for GRB blast waves in a uniform surrounding medium. Clumpiness in the surrounding medium could produce more intense neutrino emission. In our present paper, we used an analytical approach and took different parameter values, e.g., $\xi_{\mathrm{B}}=0.01$ and $n=1 \mathrm{~cm}^{-3}$ (but $\xi_{\mathrm{B}}=0.1,10^{-4}$ and $n=10^{2} \mathrm{~cm}^{-3}$ in Dermer (2002)). Our parameter values were chosen from the known afterglow fitting (e.g. Wijers \& Galama 1999; Granot et al. 1999). We derived the maximum proton energy that does not exceed $\sim 4 \times 10^{19} \mathrm{eV}$, and also considered the pion and muon cooling, all of which can affect the neutrino spectrum. We found that the detected forward-shock neutrino event rate is comparable to the reverse-shock one derived by Waxman \& Bachall (2000).

Acknowledgements. The authors thank the referee for very helpful comments to improve this paper. This work was supported by the National Natural Science Foundation of China (grants 19825109 and 19973003) and the National 973 Project (NKBRSF G19990754).

\section{References}

Blandford, R. D., \& Eichler, D. 1987, Phys. Rep., 154, 1 Blandford, R. D., \& McKee, C. F. 1976, Phys. Fluids, 19, 1130 Dai, Z. G., \& Lu, T. 2001, ApJ, 551, 249

Dermer, C. D. 2002, ApJ, 574, 65

Dermer, C., \& Humi, M. 2001, ApJ, 556, 479

Gaisser, T. K., Halzen, F., \& Stanev, T. 1995, Phys. Rep., 258, 173

Gallant, Y. A., \& Achterberg, A. 1999, MNRAS, 305, L6
Gandhi, R., Quiff, C., Reno, M., \& Sarcevic, I. 1998, Phys. Rev. D, 58, 093009

Granot, J., Piran, T., \& Sari, R. 1999, ApJ, 527, 236

Halzen, F. 1998, in Proc. of the TASI 98 Summer School, Neutrinos in Physics and Astrophysics: From $10^{-33}$ to $10^{28} \mathrm{~cm}$, Boulder, Colorado, USA, June 1998

Halzen, F. 1999, in Proc. 17th International Workshop on Weak Interactions and Neutrinos, Cape Town, South Africa

Hurley, K. 1994, Nature, 372, 652

Hillas, A. M. 1984, ARA\&A, 22, 425

Mannheim, K. 2001, AIP Conf. Proc., 558, 417

Mannheim, K., Hartmann, D., \& Funk, B. 1996, ApJ, 467, 532

Mészáros, P. 2002, ARA\&A, 40, 137

Mészáros, P., \& Rees, M. 1997, ApJ, 476, 232

Piran, T. 1999, Phys. Rep., 314, 575

Rachen, J. P., \& Mészáros, P. 1998, Phys. Rev. D, 58, 123005

Roy, M., Crawford, B. J., \& Trattner, A. 1999, in Proc. Division of Particles and Fields Conference, the University of California, Los Angeles, USA, Jan. 1999

Sari, R., \& Esin, A. A. 2001, ApJ, 548, 787

Sari, R., Piran, T., \& Narayan, R. 1998, ApJ, 497, L17

Sari, R., \& Piran, T. 1999, ApJ, 520, 641

Scully, S. T., \& Stecker, F. W. 2002, Astropart. Phys., 16, 271

Stecker, F. W. 2000, Astropart. Phys., 14, 207

van Paradijs, J., Kouveliotou, C., \& Wijers, R. A. M. J. 2000, ARA\&A, 38, 379

Vietri, M. 1995, ApJ, 453, 885

Vietri, M. 1998a, Phys. Rev. Lett., 80, 3690

Vietri, M. 1998b, ApJ, 507, 40

Waxman, E. 1995a, Phys. Rev. Lett., 75, 386

Waxman, E. 1995b, ApJ, 452, L1

Waxman, E. 2001, Nuc. Phys. B (Proc. Supp.) 91, 494

Waxman, E., \& Bahcall, J. N. 1997, Phys. Rev. Lett., 78, 2292

Waxman, E., \& Bahcall, J. N. 1999, Phys. Rev. D, 59, 23002

Waxman, E., \& Bahcall, J. N. 2000, ApJ, 541, 707

Wijers, R. A. M. J., \& Galama, T. J. 1999, ApJ, 523, 177

Zhang, B., \& Mészáros, P. 2001, ApJ, 559, 110 